Introduzindo a Geometria Fractal no Ensino Médio por meio da perspectiva de Modelagem Matemática

Francisco Geovane da Silva Araújo e Alessandra Senes Marins

\title{
INTRODUZINDO A GEOMETRIA FRACTAL NO ENSINO MÉDIO POR MEIO DA PERSPECTIVA DE MODELAGEM MATEMÁTICA
}

\author{
INTRODUCING FRACTAL GEOMETRY IN HIGHER EDUCATION \\ THROUGH THE MATHEMATICAL MODELING PERSPECTIVE
}

\author{
Francisco Geovane da Silva Araújo ${ }^{1}$ \\ Universidade Estadual Vale do Acaraú \\ Alessandra Senes Marins ${ }^{2}$ \\ Universidade Estadual Vale do Acaraú
}

\begin{abstract}
Resumo
O presente trabalho tem por objetivo apresentar uma proposta de aula para a aplicação do conceito de geometria fractal desenvolvida por meio da perspectiva de Modelagem Matemática. Para isso, realizou-se um estudo teórico e interpretativo sobre a abordagem de Modelagem Matemática e a respeito do conteúdo de geometria fractal, relacionando com a problemática escolhida, a tecelagem de um tapete. Sendo assim, percebe-se que uma aula desenvolvida sob a perspectiva de Modelagem Matemática pode proporcionar o desenvolvimento do pensamento matemático nos alunos, uma vez que ela se relaciona com situações diárias, e que a partir disso, é possível realizar ações como: a exploração de hipóteses; formulação de variáveis; uso de conhecimentos prévios; construção do modelo matemático; entre outras.
\end{abstract}

Palavras-chave: Modelagem Matemática. Ensino de Matemática. Geometria Fractal. Tecelagem.

\section{Abstract}

This paper aims to present a class proposal for the application of the concept of fractal geometry developed through the perspective of Mathematical Modeling. For this, a theoretical and interpretative study was carried out about the Mathematical Modeling approach and about the fractal geometry content, relating to the chosen problem, weaving a carpet. Thus, it is clear that a class developed from the perspective of Mathematical Modeling can provide the development of mathematical thinking in students, since it relates to daily situations, and from that, it is possible to perform actions such as: exploration of hypotheses; formulation of variables; use of prior knowledge; construction of the mathematical model; among others.

Keywords: Mathematical modeling. Mathematics teaching. Fractal geometry. Weaving.

\footnotetext{
${ }^{1}$ E-mail: franciscogeovanesilva@gmail.com.

${ }^{2}$ E-mail: ale_marins@hotmail.com.
} 
Introduzindo a Geometria Fractal no Ensino Médio por meio da perspectiva de Modelagem Matemática

Francisco Geovane da Silva Araújo e Alessandra Senes Marins

\section{Introdução}

Há tempos, no âmbito da educação, observamos muitos relatos que grande parte dos alunos apresentam dificuldades no aprendizado da matemática, sendo que parte dessas estão situadas no campo do ensino dessa disciplina. $\mathrm{O}$ ensino e a aprendizagem de qualquer disciplina, em geral, não é uma tarefa fácil, e em especial, a disciplina de matemática, pois ela promove o trabalho com: o raciocínio lógico, o pensamento crítico, a criatividade, a imaginação, são tarefas necessárias para o desenvolvimento do pensamento matemático nos alunos.

Uma questão comum no pensamento dos professores no momento de construir seus planos de aula, é a de como tornar esse momento prazeroso e significante para os alunos, e, uma forma de buscar a resposta para essa pergunta é de voltar o olhar para pesquisas na área da Educação Matemática que tratam sobre isso.

Nesse sentido, a problemática discutida nesse artigo surgiu em meio a discussões realizadas em uma disciplina do Curso de Licenciatura em Matemática da Universidade Estadual Vale do Acaraú (UVA), sobre uma abordagem de ensino em que o professor é colocado como o mediador da construção do conhecimento matemático, e o aluno o protagonista desse processo.

Dessa forma, foi levantada a seguinte pergunta de pesquisa: Como abordar o conteúdo de geometria fractal, com o uso de uma abordagem de ensino que possa promover uma aprendizagem com significados para os alunos, e que seja prazerosa e interessante?

Diante disso, foi selecionada a perspectiva de Modelagem Matemática, e desenvolvida uma aula com base nas fases relativas ao conjunto de procedimentos para a condução de uma aula sob essa abordagem de Almeida, Silva e Vertuan (2016), a fim de gerar um modelo matemático para representar a situação física escolhida, a qual utilizou uma matéria de jornal digital sobre a tecelagem, para ser trabalhada no Ensino Médio.

A escolha por essa abordagem se deu através da visão de que, além de proporcionar o ensino de conceitos matemáticos, possibilita ao aluno interpretar e ter uma visão do uso da matemática em sua realidade. Nesse sentido, segundo Bassanezi (2002, p. 16), "Modelagem Matemática consiste na arte de transformar problemas da realidade em problemas matemáticos e resolvê-los interpretando suas soluções na 
Introduzindo a Geometria Fractal no Ensino Médio por meio da perspectiva de Modelagem Matemática

Francisco Geovane da Silva Araújo e Alessandra Senes Marins

linguagem do mundo real”. Esses problemas podem ser ligados à rotina do aluno relacionando-os com os conteúdos matemáticos a serem estudados e provenientes do tema do problema.

Diante disso, o presente trabalho tem por objetivo apresentar uma proposta de aula para a aplicação do conteúdo de geometria fractal desenvolvida por meio da perspectiva de Modelagem Matemática. Nos dois próximos tópicos, apresentamos uma descrição a respeito do conteúdo de geometria fractal e sobre a abordagem escolhida para o desenvolvimento dessa proposta de aula.

\section{Geometria Fractal}

No cotidiano, deparamo-nos com figuras, objetos ou plantas que visualmente não apresentam nenhuma autossemelhança, porém ao analisarmos com mais cautela e minuciosamente os detalhes, podemos perceber que existe um determinado padrão, com dimensões fracionárias. Essas características especiais foram denominadas fractais na década de 70 pelo matemático polonês Benoit Mandelbrot.

Alguns pesquisadores do tema relatam que os fractais surgiram entre a segunda metade do séc. XIX e a primeira do séc. XX, e eram denominados na época como "Monstros Matemáticos", pois eram objetos que instigavam as noções comuns de infinito e para os quais não havia uma explicação direta (MESQUITA; MOTA, 1991).

Quanto a definição de fractal, Carvalho (2005) diz que todas as tentativas de definição deixam lacunas, tornando dessa forma um problema em aberto na matemática. E mesmo com essas lacunas, ele define fractal como, “[...] uma figura geométrica em que uma parte se assemelha a toda figura, obtida através de um processo iterativo e que pode ter uma dimensão não inteira" (CARVALHO, 2005, p. 18).

Carvalho (2005) descreve três características importantes para denominar um fractal, são elas: a autossimilaridade (exata ou aproximada), dimensão e iteração. Dessa forma, podemos citar como exemplo de fractais: o Floco de Neve de Koch; Triângulo de Sierpinski, Curva de Peano, Conjuntos de Mandelbrot, dentre outros. Além disso, os fractais podem ser aplicados em diversas áreas, como no Ensino da matemática, Geologia, Biologia, Medicina, Informática, Linguística, Meteorologia, etc.

É pouco comum professores abordarem a geometria fractal no ensino e na aprendizagem da matemática, isso pode ocorrer pelo fato de não se sentirem 
Introduzindo a Geometria Fractal no Ensino Médio por meio da perspectiva de Modelagem Matemática

Francisco Geovane da Silva Araújo e Alessandra Senes Marins

confortáveis ao ensinar esse tema, pois a busca por diferentes ferramentas que podem auxiliar na aprendizagem dos alunos demanda uma maior dedicação, estudo e planejamento. Para Barbosa (2005) o estudo da geometria fractal em sala de aula, propicia aos estudantes compreender a matemática e seus significados, como

[...] pode-se explorar: o floco de neve e a curva de Koch; triângulo e tapete de Sierpinski, conduzindo o aluno a refletir e observar o senso estético presente nessas entidades geométricas, estendendo para as suas propriedades, através da regularidade harmoniosa nas suas próprias irregularidades (BARBOSA, 2005, p. 14).

Nessa perspectiva, podemos supor que o aluno ao se deparar com este conceito, poderá associar a matemática com os objetos de formas igualmente complexas encontradas em seu cotidiano, despertando assim uma visão mais reflexiva e ampla sobre as similaridades presentes nos objetos.

\section{Modelagem Matemática como abordagem de ensino}

Ao planejar uma aula que seja interessante e proporcione significados matemáticos e da realidade, e que considere possíveis dificuldades e conhecimentos prévios dos alunos, alguns professores buscam por diferentes abordagens para o ensino da Matemática.

Nesse sentido, uma perspectiva de ensino que vem se destacando no campo da Educação Matemática, é a de Modelagem Matemática. Para Bassanezi (2002, p. 16), essa abordagem “[...] consiste, essencialmente, na arte de transformar problemas da realidade em problemas matemáticos e resolvê-los interpretando suas soluções na linguagem do mundo real”.

Segundo Almeida, Silva e Vertuan (2016, p. 15), a "Modelagem Matemática visa propor soluções para problemas por meio de modelos matemáticos. O modelo matemático, nesse caso, é o que 'da forma' à solução do problema e a Modelagem Matemática é a 'atividade' de busca por essa solução".

E de acordo com Barbosa (2001, p. 6) o ensino de matemática desenvolvido sob essa perspectiva proporciona “[...] um ambiente de aprendizagem no qual os alunos são convidados a indagar e/ou investigar, por meio da Matemática, situações oriundas de outras áreas da realidade".

Além disso, os Parâmetros Curriculares Nacionais do Ensino Médio - PCNEM (BRASIL, 2006, p. 69) propõe “[...] que os alunos saibam usar a Matemática para 
Introduzindo a Geometria Fractal no Ensino Médio por meio da perspectiva de Modelagem Matemática

Francisco Geovane da Silva Araújo e Alessandra Senes Marins

resolver problemas práticos do cotidiano; para modelar fenômenos em outras áreas do conhecimento e que percebam a Matemática como um conhecimento social e historicamente construído", o que vai de encontro com a proposta da perspectiva de Modelagem Matemática.

Corroborando com os PCN, Franchi (2007) ressalta que a Modelagem Matemática, deve fazer parte dos currículos de matemática, pois possibilita aos estudantes trabalhar com a aplicação da Matemática, com a construção de modelos, e a partir deles resolver problemas. Nesse sentido, há necessidade de a organização curricular privilegiar espaços de "[...] investigação, reflexão, cooperação e construção de conhecimento" (FRANCHI, 2007, p. 179).

Tendo em vista esses apontamentos sobre essa perspectiva, é notório que o tema abordado se relaciona com o cotidiano dos estudantes e que por meio dessa abordagem, é possível encontrar soluções para diversos problemas de nossa sociedade.

Sendo assim, entende-se que a Modelagem Matemática torna-se pertinente no processo de ensino e de aprendizagem da matemática, em que essa disciplina é ensinada de modo relevante para os alunos, levando em consideração a realidade educacional.

Diante disso, Almeida, Silva e Vertuan (2016) apresentam algumas fases para o desenvolvimento de uma atividade de Modelagem Matemática em sala de aula, em que considere o cotidiano e aspectos externos à matemática e que parta de uma situação problemática, que são:

- Interação ${ }^{3}$ : É o primeiro momento, em que os alunos irão conhecer a situação problema, desde dados quantitativos ou qualitativos por meio do contato direto ou indireto. É a etapa de familiarização do estudante com o assunto a ser modelado;

- Matematização: É a fase em que ocorre a transformação da linguagem natural para a linguagem matemática. Nessa, também acontece a identificação de variáveis e a formulação das hipóteses;

- Resolução: Essa etapa sucede a construção do modelo matemático, em que serão respondidas as perguntas formuladas anteriormente;

- Interpretação de Resultados e Validação: É o momento de avaliação do modelo encontrado, que consiste em conferir se os dados apresentados no início

\footnotetext{
${ }^{3}$ Interação da modelagem diferenciar da iteração do tapete.
} 
Introduzindo a Geometria Fractal no Ensino Médio por meio da perspectiva de Modelagem Matemática

Francisco Geovane da Silva Araújo e Alessandra Senes Marins

da atividade são compatíveis. Nessa fase, é necessário que leve em consideração

o grau de aproximação dos resultados encontrados com o modelo obtido em

relação aos dados fornecidos anteriormente. Em muitos dos casos, os resultados

obtidos são muito próximos.

Além disso, conforme esses autores, essas fases podem não ocorrer de forma linear, são permitidas "idas e vindas" entre elas (ALMEIDA; SILVA; VERTUAN, 2016). Diante disso, considerando os aspectos citados sobre essa perspectiva de ensino, e utilizando as fases de Modelagem Matemática conforme Almeida, Silva e Vertuan (2016), apresentamos a proposta de aula envolvendo essa abordagem intitulada de "Tecelagem de tapete e matemática, uma possível relação".

\section{Modelagem Matemática: uma proposta de ensino}

A atividade está direcionada para alunos do $3^{\underline{0}}$ ano do Ensino Médio, e os requisitos necessários para essa prática são os conteúdos de potenciação, área do quadrado e sequências geométricas. Assim, a aula consiste em apresentar o estudo de geometria fractal através da metodologia Modelagem Matemática, buscando tornar a aula agradável a partir de uma situação problema inicial, chegando a um modelo matemático por meio da fórmula matemática utilizada para descobrir quantos quadrados de determinada cor o tapete possui, e respectivamente suas áreas.

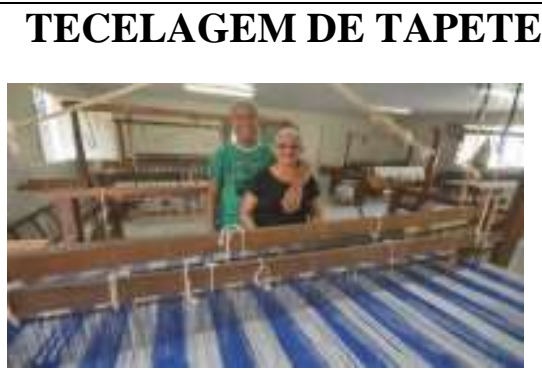

Foto: Assis Fernandes/O Dia

\section{Arte milenar, a tecelagem continua sendo fonte de renda}

Tecelãs descrevem como a atividade continua gerando lucros, mesmo diante dos desafios impostos pela desvalorização e avanço tecnológico.

Um trabalho delicado e minucioso, feito à mão, que requer atenção e cuidado. Mais que entrelaçar fios, tear é uma arte milenar que entrelaça vidas. Uma atividade que, por muito tempo, foi passada por gerações, mas tem se perdido ao longo dos anos, principalmente pela falta de interesse dos mais jovens.

A tecelagem está presente na vida de dona Maria do Carmo Silva Santos, de 67 anos, desde quando ela ainda estava na barriga de sua mãe, no município de Pedro II. E assim foi por muitas gerações passadas, quando as mulheres da família produziam redes.

"Na minha casa, eu tenho um tear de parede e todo dia eu faço uma coisinha de um tapete. Eu, mesmo quase cega, coloco meus óculos e faço minhas peças. Eu gosto do que faço e me mantenho com isso", conta a tecelã. 
Introduzindo a Geometria Fractal no Ensino Médio por meio da perspectiva de Modelagem Matemática

Francisco Geovane da Silva Araújo e Alessandra Senes Marins

Dona Maria do Carmo se emociona ao descrever essa como a atividade mais importante de sua vida e por temer que a tecelagem, como é produzida na Associação, desapareça por falta de interesse das pessoas em trabalhar com artesanato.

"Isso aqui é tudo para mim. Foi isso que eu aprendi desde pequena, então é minha vida e eu dou maior valor. A Associação ainda existe por causa de mim e da Raimunda, que vem todo dia aqui ver como estão indo as coisas, porque temos medo que se acabe", conta.

Fonte: Portal o dia (2018).

\subsection{Interação}

Uma forma muito interessante de se produzir belos tapetes decorativos utilizando a matemática é pelo processo de autossemelhança, no qual se denominam como fractais. Informalmente, dizemos que uma figura é autossemelhante se partes dessa figura são semelhantes à figura vista como um todo. Um jovem tecelão instigado com a história de Dona Maria do Carmo percebeu que seria possível fazer um tapete diferenciado com autossemelhança criado por um processo recursivo, resolveu então produzí-lo da seguinte forma:

- Etapa 1: Considere os fios no formato de um tapete quadrado de $27 \mathrm{~cm}$ de lado representado por (l), formando uma área de $729 \mathrm{~cm}^{2}$ de cor única (preto) que será dividido em nove quadrados idênticos (Iteração 1). Inicia-se o processo tecendo de vermelho o quadrado central, restando 8 quadrados pretos (Iteração 1).

- Etapa 2: Repete-se o processo com cada um dos quadrados restantes, ou seja, divide-se cada um deles em 9 quadrados idênticos tecendo o quadrado central de cada um de vermelho, restando apenas os quadrados pretos (Iteração 2).

- Etapa 3: Repete-se a etapa 2,como apresentada a seguir:

Figura 1- Processo de iterações no tapete de Sierpinski.

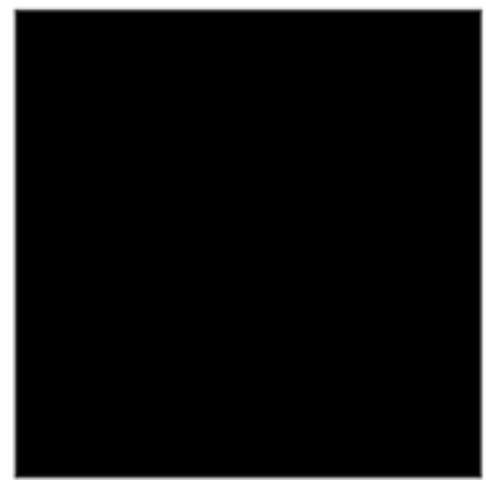

Iteração 0

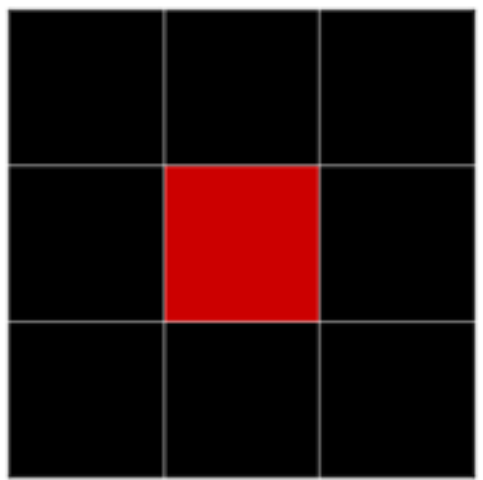

Iteração 1

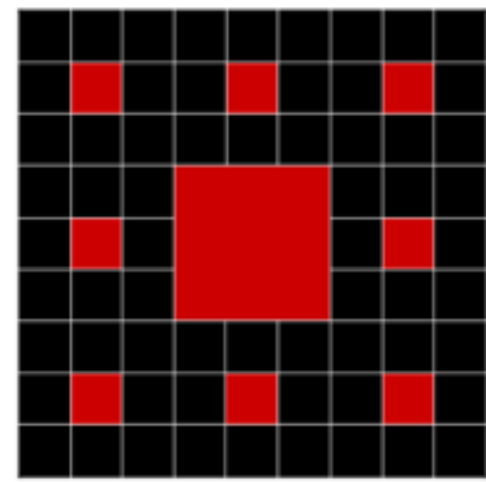

Iteração 2

Fonte: Elaborada pelos autores (2019). 
Introduzindo a Geometria Fractal no Ensino Médio por meio da perspectiva de Modelagem Matemática

Francisco Geovane da Silva Araújo e Alessandra Senes Marins

O jovem decidiu por algum motivo parar esse processo na iteração 4, na qual percebeu que o tapete já estava bonito, além disso, notou que havia um padrão que o levou até ali. Nesse momento, decidiu verificar quantos quadrados vermelhos continham no tapete e quantos $\mathrm{cm}^{2}$ de preto foram usados na tecelagem desse tapete.

\subsection{Matematização}

Determinaremos nomes para as problemáticas, para termos assim uma melhor compreensão.

\section{Problemática Alfa}

- Problemática Alfa: Descobrir quantos quadrados vermelhos contém no tapete?

- Hipóteses: As iterações relacionam-se com a quantidade de quadrados vermelhos.

- Variáveis: Iteração $(x)$ - variável independente; Quantidade de quadrados menores vermelhos $(y)$ - variável dependente.

\section{Problemática Beta}

- Problemática Beta: Quantos $\mathrm{cm}^{2}$ de preto foram usados na tecelagem desse tapete?

- Hipóteses:A área dos quadrados pretos relaciona-se com a quantidade de iterações.

- Variáveis: Iteração $(x)$ - variável independente. Área dos quadrados vermelhos $(z)$ - variável dependente.

\subsection{Resolução}

A priori, não sabemos como estes quadrados comportam-se, o que eles descrevem. Sendo assim, representaremos em uma tabela, para percebermos se ocorre algum tipo de padrão conhecido. Assim, partiremos para as resoluções de acordo com as problemáticas Alfa e Beta.

\section{Problemática Alfa:}

Tabela 1: Cálculo da quantidade de quadrados vermelhos após as iterações.

\begin{tabular}{|c|c|}
\hline Iterações $(\mathrm{x})$ & $\mathrm{N}^{\mathrm{o}}$ de quadrados menores tecido de vermelho $(\mathrm{y})$ \\
\hline 1 & 1 \\
\hline 2 & 8 \\
\hline 3 & 64 \\
\hline.. &.. \\
\hline
\end{tabular}


Introduzindo a Geometria Fractal no Ensino Médio por meio da perspectiva de Modelagem Matemática

Francisco Geovane da Silva Araújo e Alessandra Senes Marins

\begin{tabular}{|c|c|}
\hline $\mathrm{x}$ & $8^{x-1}$ \\
\hline
\end{tabular}

Fonte: Elaborada pelos autores (2019).

\section{Modelo Alfa:}

Para descobrimos a quantidade de quadrados menores vermelhos que contém na iteração 4, bastar substituir os dados: $x=4$ iteração:

$$
8^{4-1}=512 \text { quadrados menores vermelhos }
$$

Podemos perceber que $\boldsymbol{I}(\boldsymbol{x})=\boldsymbol{I}(\mathbf{1}) \times q^{x-1}$; onde $q$ é a razão dessa Progressão Geométrica, obtendo a fórmula do n-ésimo termo de uma PG.

$$
I(x)=I(1) * q^{x-1} \rightarrow a_{n}=a_{1} \times q^{n-1}
$$

$I(x)=a_{n}=n$-ésimo termo de uma PG;

$I(1)=a_{1}=$ Primeiro termo de uma PG;

$q=$ Razão de uma PG.

E para descobrimos o número de quadrados tecidos de vermelho, basta somar todos os quadrados vermelhos das iterações:

$$
1+8+64+512=585 \text { quadrados vermelhos. }
$$

\section{Problemática Beta:}

Para encontrarmos a quantidade de tecido preto em $\mathrm{cm}^{2}$, calcularemos primeiro a quantidade de $\mathrm{cm}^{2}$ tecido de vermelho. Considerando sendo $l=27 \mathrm{~cm}$ o lado do tapete, logo sua área inicial será $l^{2}=729 \mathrm{~cm}^{2}$, assim temos a seguinte tabela:

Tabela 2: Cálculo da área dos quadrados vermelhos após as iterações.

\begin{tabular}{|c|c|c|c|c|}
\hline Iterações (x) & $\begin{array}{c}\mathrm{N}^{\mathrm{o}} \text { de quadrados } \\
\text { menores tecido } \\
\text { de vermelho }\end{array}$ & $\begin{array}{c}\mathrm{N}^{\circ} \text { de quadrados } \\
\text { divididos } \\
\text { Modelo Beta 1 }\end{array}$ & $\begin{array}{c}\text { Área do } \\
\text { menor } \\
\text { quadrado } \\
\text { vermelho (z) }\end{array}$ & $\begin{array}{c}\text { Área dos } \\
\text { quadrados } \\
\text { vermelhos }\end{array}$ \\
\hline 1 & 1 & $\frac{l^{2}}{9}$ & 81 & 81 \\
\hline 2 & 8 & $\frac{l^{2}}{81}$ & 9 & 72 \\
\hline
\end{tabular}

Boletim Cearense de Educação e História da Matemática - Volume 06, Número 18, 21 - 34 (2019) DOI: 10.30938/bocehm.v6i18.2333 
Introduzindo a Geometria Fractal no Ensino Médio por meio da perspectiva de Modelagem Matemática

Francisco Geovane da Silva Araújo e Alessandra Senes Marins

\begin{tabular}{|c|c|c|c|c|}
\hline 3 & 64 & $\frac{l^{2}}{729}$ & 1 & 64 \\
\hline 4 & 512 & $\frac{l^{2}}{6561}$ & $\frac{1}{9}$ & $\frac{512}{9}$ \\
\hline.. &.. &.$\cdot$ &.. &.$\cdot$ \\
\hline $\mathrm{x}$ & $8^{x-1}$ & $\frac{l^{2}}{9^{x}}$ & 0 & 0 \\
\hline
\end{tabular}

Fonte: Adaptada de Vielmo e Dalberto (2013).

Analisando as figuras da situação problemas, podemos observar que:

- A área do quadrado da $1^{\text {a }}$ iteração será um terço do quadrado de seu lado;

- A área do quadrado da $2^{a}$ iteração será um nono do quadrado de seu lado;

- A área do quadrado da $3^{\mathrm{a}}$ iteração será um vinte sete avos do quadrado de seu lado.

Preenchendo dessa forma a tabela de acordo com as iterações. Sendo $l^{2}=729 \mathrm{~cm}^{2}$, e como parou na iteração 4 então temos $x=4$.

Substituindo no Modelo Beta 1 para encontrarmos a área do menor quadrado vermelho, temos:

$$
\frac{l^{2}}{9^{x}}=\frac{729}{9^{4}}=\frac{729}{6561}=\frac{1}{9} \approx 0,0111 \mathrm{~cm}^{2}
$$

Como descobrimos a área do menor quadrado vermelho na iteração 4, basta multiplicarmos por suas respectivas quantidades e depois fazermos o somatório:

$$
\begin{aligned}
& \left(81 \cdot 1 ; 9 \cdot 8 ; 1 \cdot 64 ; 529 \cdot \frac{1}{9}\right)=\left(81+72+64+\frac{529}{9}\right)= \\
& =273,88 \mathrm{~cm}^{2} \text {, a área dos quadrados vermelhos. }
\end{aligned}
$$

Basta agora subtrair a área dos quadrados vermelhos pela área total do quadrado.

$$
729-273,88=455,12 \mathrm{~cm}^{2} \text { de tecido preto. }
$$

\section{Modelo Beta 2}

Analisando a área do tecido preto da figura 1 em cada iteração. Inicialmente, temos um quadrado de lado $l$ na iteração 0 , logo sua área é:

$$
A_{0}=l^{2}
$$


Introduzindo a Geometria Fractal no Ensino Médio por meio da perspectiva de Modelagem Matemática

Francisco Geovane da Silva Araújo e Alessandra Senes Marins

Como a figura é dividida em nove quadrados menores na iteração 1 , temos que a área da figura é:

$$
A_{1}=\frac{8}{9} A_{0}
$$

Já na iteração 2, teremos cada um dos quadrados pretos da iteração anterior dividido do mesmo modo:

$$
A_{2}=\frac{8}{9}\left(\frac{8}{9} A_{0}\right)
$$

Seguindo esse processo para as demais iterações, podemos percebemos a construção de um modelo. Assim, na iteração $n$ a área da figura será:

$A_{n}=\left(\frac{8}{9}\right)^{n} A_{0}$

E fazendo as substituições, temos que a área do tecido preto na iteração 4 é:

$A_{0}=l^{2}=27^{2}=729 \mathrm{~cm}^{2}$

$A_{4}=\left(\frac{8}{9}\right)^{4} 729$

$$
A_{4}=455,11 \mathrm{~cm}^{2} \text { tecido de preto }
$$

O resultado foi semelhante ao anterior, assim fica a critério do aluno a escolha do modelo, válido ressaltar que encontramos uma Progressão Geométrica de razão $q=\frac{1}{9}$. Podendo ser resolvido pelo Modelo Alfa encontrado para a Problemática Alfa.

\subsection{Interpretação de Resultados e Validação}

Utilizou-se de estratégias de resolução distintas, contudo, chegamos a modelos diferentes, cabendo fazermos uma análise dos dados que estes fornecem, de forma a encontrarmos o modelo que satisfaça as informações do problema. A validação dos

dados pode ser realizada nos Softwares Excel e Geogebra. Considerando $q=\frac{1}{9}$ no Modelo Alfa e $A_{0}=729 \mathrm{~cm}^{2}$ no Modelo Beta 2, conforme a tabela 3 temos: 
Introduzindo a Geometria Fractal no Ensino Médio por meio da perspectiva de Modelagem Matemática

Francisco Geovane da Silva Araújo e Alessandra Senes Marins

Tabela 3: Validação dos dados no Software Excel.

\begin{tabular}{|c|c|c|c|}
\hline Iterações I(x) & $\begin{array}{c}\text { Modelo Alfa } \\
I(x)=I(1) \times q^{x-1}\end{array}$ & Área & $\begin{array}{c}\text { Modelo Beta 2 } \\
A_{n}=\left(\frac{8}{9}\right)^{n} \cdot A_{0}\end{array}$ \\
\hline $\mathrm{I}(0)$ & 729 & $A_{0}$ & 729 \\
\hline $\mathrm{I}(1)$ & 648 & $A_{1}$ & 648 \\
\hline $\mathrm{I}(2)$ & 576 & $A_{2}$ & 576 \\
\hline $\mathrm{I}(3)$ & 512 & $A_{3}$ & 512 \\
\hline $\mathrm{I}(4)$ & 455,11 & $A_{4}$ & 455,11 \\
\hline
\end{tabular}

Fonte: Elaborada pelos autores (2019).

Analisando a tabela, podemos observar que os dois modelos mostram os resultados exatamente iguais. Para validar os modelos utilizando o Software Geogebra, temos:

Figura 2 - Tapete de Sierpinski construído no Softaware Geogebra com os valores das áreas tecidos de preto em cada iteração.

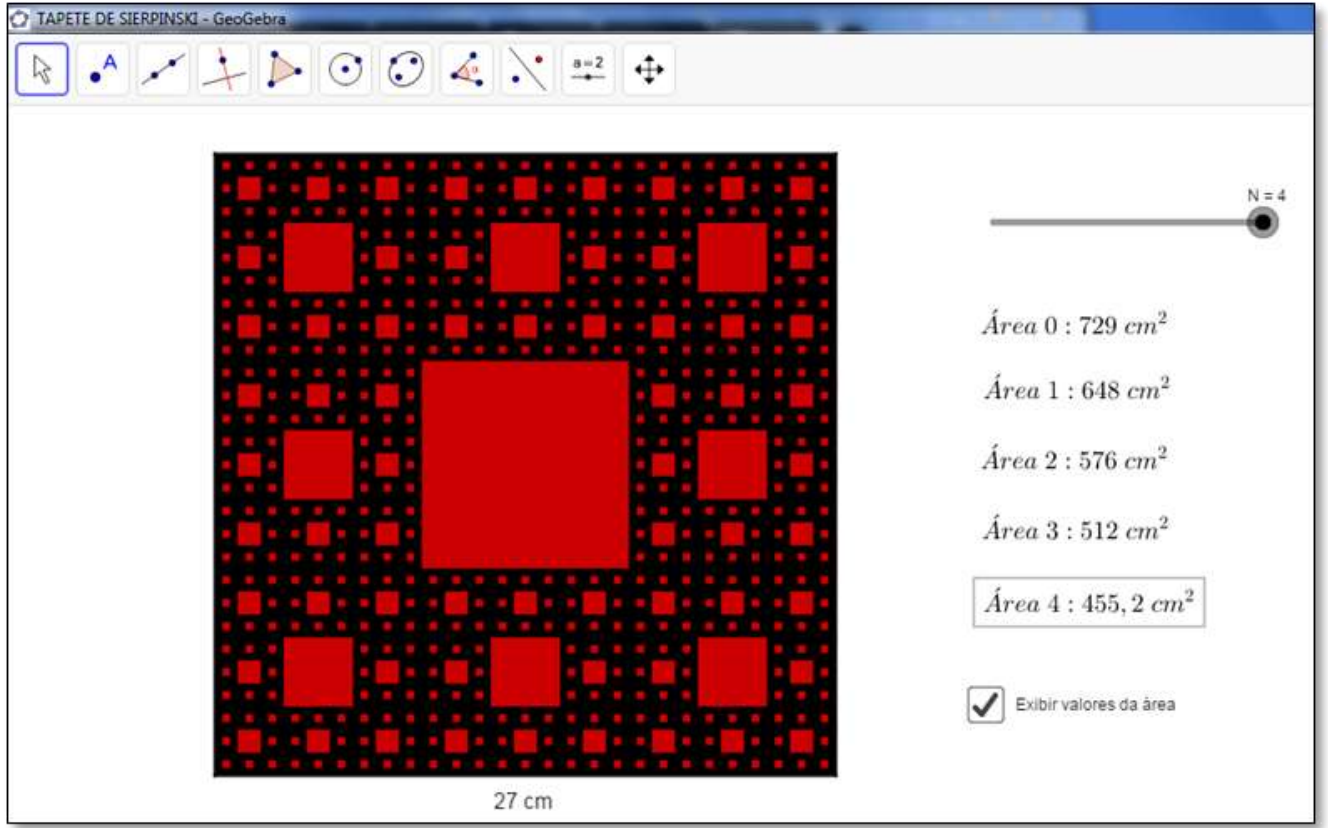

Fonte: Elaborada pelos autores (2019).

Podemos observar na figura 2 que os valores são iguais os da tabela 3, somente a Área 4 que difere nos décimos devido ao arredondamento do software. Logo, a 
Introduzindo a Geometria Fractal no Ensino Médio por meio da perspectiva de Modelagem Matemática

Francisco Geovane da Silva Araújo e Alessandra Senes Marins

validação do modelo matemático obtido pelo uso dos softwares que será observada pelos alunos, dará as devidas definições matemáticas.

\section{Considerações finais}

Ao concluirmos o presente artigo, entendemos que o uso de diferentes abordagens de ensino é importante para a construção do pensamento matemático para os estudantes, proporcionando dinâmicas de aula não habituais que colocam o aluno como autor do desenvolvimento da sua aprendizagem matemática.

Nesse sentido, esse trabalho apresentou uma forma de se utilizar a abordagem de Modelagem Matemática no estudo da geometria fractal, promovendo uma aula criativa e dinâmica, partindo de um problema, em princípio, não matemático, permitindo a exploração de hipóteses, formulação de variáveis e construção do modelo matemático, e finalizando com as definições do objeto matemático em questão.

Com base no estudo dessa perspectiva, entendemos que ao utilizá-la aliada a um planejamento detalhado, considerando objetivos em relação ao conteúdo e ações didático-pedagógicas que contribuem para a gestão da aula, é possível promover a construção do conhecimento matemático nos alunos, fazendo com que reconheçam que essa disciplina está inserida em vários campos do conhecimento.

Contudo, o professor precisa desenvolver um olhar para promover a exploração em sala de aula, possibilitando que seus alunos investiguem a problemática em questão e utilizem de conhecimentos prévios no desenvolvimento da aula.

\section{Referências}

ALMEIDA, L. M. W.; SILVA, K. P.; VERTUAN, R. E. Modelagem Matemática na educação básica. São Paulo: Contexto, 2016.

BARBOSA, J. C. Modelagem Matemática e os professores: a questão da formação. Bolema: Boletim de Educação Matemática, Rio Claro, n. 15, p. 5-23, 2001.

BARBOSA, R. M. Descobrindo a geometria fractal para sala de aula. $2^{\circ}$ ed. Belo Horizonte: Autêntica, 2005.

BASSANEZI, R. C., Ensino-Aprendizagem com Modelagem Matemática. Editor Contexto, 2002.

BRASIL. Ministério da Educação-MEC. Secretaria de Educação Básica. Orientações 
Introduzindo a Geometria Fractal no Ensino Médio por meio da perspectiva de Modelagem Matemática

Francisco Geovane da Silva Araújo e Alessandra Senes Marins

Curriculares para o Ensino Médio: Ciências da Natureza, Matemática e suas

Tecnologias. Brasília, 2006.

CARVALHO, H. C. Geometria Fractal: perspectivas e possibilidades no ensino de matemática. 2005. 101 f. Dissertação (Mestrado em Educação) - Curso de Pós-

Graduação em Ensino em Ciência e Matemática, Universidade Federal do Pará, Belém, 2005. Disponível em:

http://repositorio.ufpa.br/jspui/bitstream/2011/1857/1/Dissertacao_GeometriaFractalPer pectivas.pdf. Acesso em 02 de janeiro de 2019.

Clube Militar Naval 1991. Disponível em:

http://www.albertomesquita.net/am/fractais/fractais1.htm. Acesso em 02 de janeiro de 2019.

FRANCHI, R. H. de O. Ambientes de Aprendizagem fundamentados na modelagem matemática e na informática como possibilidades para a Educação Matemática. In: BARBOSA, J. C.; CALDEIRA, A. D.; ARAÚJO, J. L. de. Modelagem Matemática na Educação Matemática Brasileira: pesquisas e práticas educacionais. Recife: SBEM, 2007.

LOPES, I. Arte milenar, a tecelagem continua sendo fonte de renda. Jornal O DIA. Disponível em: https://www.portalodia.com/noticias/piaui/arte-milenar,-a-tecelagemcontinua-sendo-fonte-de-renda-316414.html. Acesso em: 02 de janeiro de 2019.

MESQUITA, A.; MOTA, M. G., Fractais - A linguagem do caos. In. Anais do

VIELMO, S. E.; DALBERTO, F. Abordagem do ensino de matemática através de fractais e recursos tecnológicos. In:VI Congresso Internacional de Ensino de Matemática-2013. 2013. Disponível em:http://www.conferencias.ulbra.br/index.php/ciem/vi/paper/viewFile/797/390. Acesso em 02 de janeiro de 2019. 